\title{
Sister Mary Joseph nodule caused by metastatic desmoplastic small round cell tumor: A clinicopathological report
}

\author{
NOPPADOL LARBCHAROENSUB ${ }^{1}$, ATCHARAPORN PONGTIPPAN ${ }^{1}$, DUANGJAI PANGPUNYAKULCHAI ${ }^{1}$, \\ SITH PHONGKITKARUN ${ }^{2}$, PANUWAT LERTSITHICHAI ${ }^{3}$ and THITIYA S. DEJTHEVAPORN ${ }^{4}$ \\ Departments of ${ }^{1}$ Pathology, ${ }^{2}$ Radiology, ${ }^{3}$ Surgery and ${ }^{4}$ Medicine, \\ Faculty of Medicine Ramathibodi Hospital, Mahidol University, Ratchathewi, Bangkok 10400, Thailand
}

Received May 25, 2016; Accepted June 28, 2016

DOI: $10.3892 /$ mco.2016.1002

\begin{abstract}
Sister Mary Joseph nodule is an uncommon metastatic intra-abdominal malignancy involving the umbilicus. The present study describes a rare case of desmoplastic small round cell tumor (DSRCT), histological grade 3 , high grade, Gilly classification 4, stage IV, in an 18-year-old Thai man presenting with the Sister Mary Joseph nodule, ascites and pleural effusion. The histopathological examination of the umbilical mass revealed the presence of malignant small round cells associated with prominent stromal desmoplasia. Immunohistochemical stains showed positive reactivity to cytokeratin, desmin, neuron-specific enolase, Wilms' tumor 1, CD56, CD99 and SWI/SNF-related matrix-associated actin-dependent regulator of chromatin subfamily B member 1 (SMARCB1)/INI1 in the small round cells. Fine needle aspirations of the ascitic fluid and pleural effusion were performed, and immunocytochemistry revealed a metastatic DSRCT. The patient received a VDC/IE regimen of chemotherapy, comprising vincristine, doxorubicin, and cyclophosphamide alternating with ifosfamide and etoposide; however, the patient developed systemic metastasis and succumbed to the disease 6 months later.
\end{abstract}

\section{Introduction}

Desmoplastic small round cell tumor (DSRCT) is an uncommon malignant mesenchymal tumor demonstrating a complex pattern of simultaneous polyphenotypic differentiation, expressing proteins associated with epithelial, muscular and neural differentiation (1). Its various appellations include

Correspondence to: Dr Noppadol Larbcharoensub, Department of Pathology, Faculty of Medicine Ramathibodi Hospital, Mahidol University, 270 Rama VI Road, Ratchathewi, Bangkok 10400, Thailand

E-mail: noppadol.lar@mahidol.ac.th

Key words: desmoplastic small round cell tumor, Sister Mary Joseph nodule, umbilical metastasis, ascitic fluid, pleural effusion, adult intra-abdominal desmoplastic small round cell tumor, intra-abdominal desmoplastic small cell tumor with divergent differentiation, polyphenotypic small round cell tumor and mesothelioblastoma (1). DSRCT was first described by Gerald and Rosai in 1989 (2). DSRCT has a highly aggressive clinical course with high risk of local recurrence and distant metastases, and is automatically assigned as high-grade sarcoma. The patterns of metastasis of DSRCT are similar to those of the other abdominal malignancies such as gastrointestinal carcinoma, with both intraperitoneal and retroperitoneal lymphatic routes being frequently encountered (1). The overall prognosis is poor due to the aggressive nature of the disease. Despite aggressive treatment, the 5-year survival rate is $<15 \%$ (3).

Sister Mary Joseph nodule is a rare and peculiar physical sign that is encountered in 1-3\% of patients with intra-abdominal malignancy (4). It is an umbilical intraperitoneal metastasis from an underlying extensive intra-abdominal malignancy. Commonly encountered primary tumors associated with umbilical metastasis include stomach, ovary, colorectum and pancreas. Umbilical metastasis presenting as Sister Mary Joseph nodule from DSRCT is extremely rare (5-7). The present study reports a rare case of Sister Mary Joseph nodule caused by metastatic DSRCT.

\section{Case report}

Clinical summary. An 18-year-old Thai man presented with an umbilical nodule and a gradual enlargement of his abdominal mass for 4 months. The patient had no history of significant illness in the past. The patient did not drink alcohol or smoke, and had no history of tuberculosis and cancer among his family members. Physical examination revealed a well-defined, firm violaceous mass, measuring $2.3 \mathrm{~cm}$ in its greatest dimension, located at the umbilicus (Fig. 1). There was neither superficial vein dilatation nor evidence of inflammation. The abdomen exhibited an ill-defined firm mass measuring $15 \times 13 \times 10 \mathrm{~cm}$. The cervical and inguinal lymph nodes could not be palpated. An evaluation of the right lung revealed decreased breath sound. Computed tomography (CT, Multidetector CT: Aquilion CX, Toshiba Medical Systems Corporation, Tokyo, Japan) of the abdomen revealed a diffuse peritoneal mass (Fig. 2) and extensive intraperitoneal seeding, and multiple intra-abdominal, pelvic and bilateral inguinal lymphadenopathies, with multiple 
bilobar hepatic metastases. Left ureteric obstruction with hydronephrosis and partial colonic obstruction were detected. The patient underwent a fine needle aspiration (FNA) of the ascitic fluid and right pleural effusion, and a minilaparotomy with incisional biopsy of the intra-abdominal mass and Sister Mary Joseph nodule. The pathological diagnosis was DSRCT, histological grade 3, high grade, Gilly classification 4, classified as stage IV. The patient achieved a stable disease following four courses of VDC/IE regimen of chemotherapy, which consisted of vincristine $\left(1.4 \mathrm{mg} / \mathrm{m}^{2}\right)$, doxorubicin $\left(75 \mathrm{mg} / \mathrm{m}^{2}\right)$ and cyclophosphamide $\left(1,200 \mathrm{mg} / \mathrm{m}^{2}\right)$ alternating with ifosfamide $\left(9 \mathrm{gm} / \mathrm{m}^{2}\right)$ and etoposide $\left(500 \mathrm{mg} / \mathrm{m}^{2}\right)$. The patient desired no further treatment. Finally, the patient succumbed to the disease 6 months following the diagnosis of DSRCT with systemic metastasis. No autopsy was performed.

The present study was approved by the Committee on Human Rights Related to Researches involving Human Subjects (Faculty of Medicine, Ramathibodi Hospital, Mahidol University; ID05-51-32).

Pathological findings. FNA of the ascitic fluid and right pleural effusion revealed cohesive groups of tumor cells, revealing the presence of small-sized cells with round, slightly pleomorphic nuclei with inconspicuous nucleoli, and a moderate amount of cytoplasm without definitive differentiation. The histopathology of the intra-abdominal mass and Sister Mary Joseph nodule revealed malignant small, round blue cells embedded in a dense desmoplastic stroma (Fig. 3A and B). The cells exhibited vesicular nuclei, with scant cytoplasm and numerous mitoses. Neither a glandular structure nor rosette formation was detected. The intervening stroma was negligible in several areas, or abundant and densely collagenous in others. No vascular proliferation was identified. There were numerous tumor emboli in the lymphatic channels. The immunocytohistochemical stains for cytokeratin (AE1/AE3) (clone AE1/3; 1/100 dilution), epithelial membrane antigen (EMA) (clone E29; 1/100 dilution), desmin (clone D33; 1/200 dilution) (all from Dako, Carpinteria, CA, USA), neuron-specific enolase (NSE) (clone 5E2; 1/100), Wilms' tumor 1 (WT1) (clone WT49; 1/40) (both from Leica, Mannheim, Germany), vimentin (clone Vim3B4; 1/200 dilution; Dako), CD56 (clone 1B6; 1/200; Leica), CD99 (clone 12E7; 1/75; Dako) and SWI/SNF-related matrix-associated actin-dependent regulator of chromatin subfamily B member 1 (SMARCB1/INI1) (clone MRQ-27, optimally diluted; Ventana Medical Systems, Inc., Tucson, AZ, USA) were positive in the malignant small round cells (Fig. 3C and D). The MIB-1 (polyclonal; 1/100; Dako) index was $80 \%$. The tumor cells were immunonegative for chromogranin A (clone DAK-A3; 1/100), synaptophysin (polyclonal; 1/100), leukocyte common antigen (LCA) (clone 2B11; 1/100), CD3 (clone F7.2.38; 1/50) and CD20 (clone L26; 1/200) (all from Dako).

\section{Discussion}

DSRCT is an uncommon malignant mesenchymal neoplasm composed of small round tumor cells associated with prominent stromal desmoplasia and polyphenotypic differentiation. The average age at presentation occurs principally during the second to third decade, with a range of 6 to 54 years $(1,8)$.

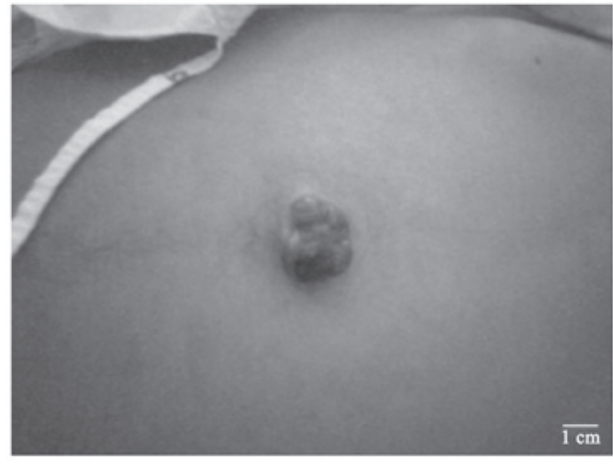

Figure 1. Physical examination of the abdomen revealed a well-defined mass measuring $2.3 \mathrm{~cm}$ in diameter, locating at the umbilicus.

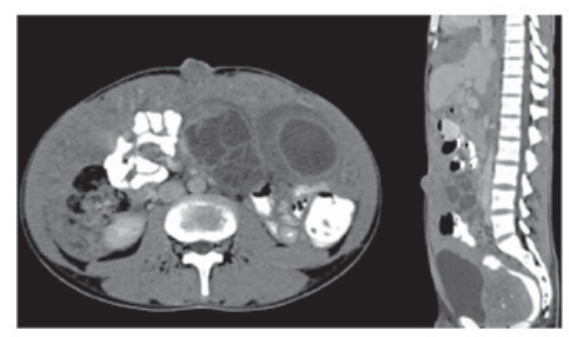

Figure 2. CT scans of the abdominal area. Left: Axial CT scan of the abdomen demonstrated a $2.3 \mathrm{~cm}$ soft tissue nodule at the umbilicus. Right: Sagittal CT scan of the abdomen at the midline demonstrated the umbilical nodule and diffuse peritoneal mass fill-in the entire peritoneal cavity. CT, computed tomography.

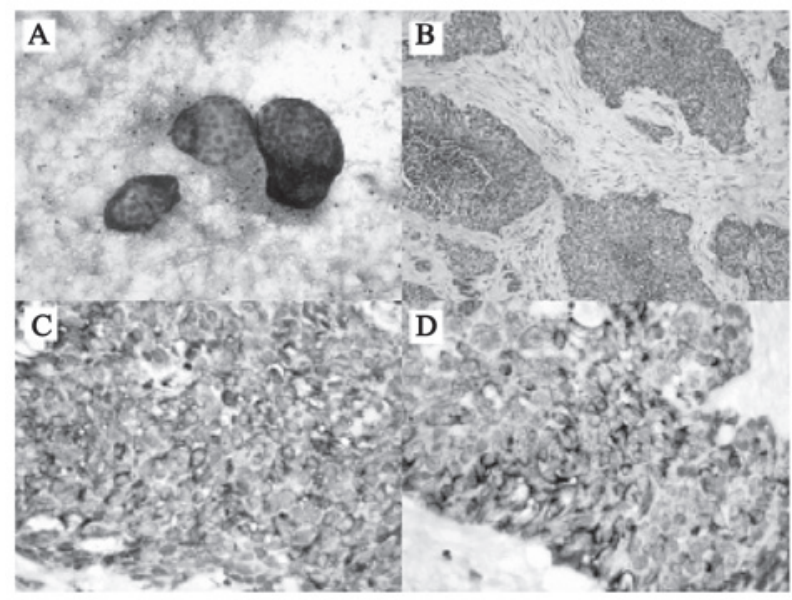

Figure 3. FNA of the right pleural effusion. (A) Cohesive groups of tumors are shown, revealing small sized cells with round, slightly pleomorphic nuclei with inconspicuous nucleoli and a moderate amount of cytoplasm (Papanicolaou's stain; magnification, x200). (B) Histopathology of Sister Mary Joseph nodule revealed malignant small round cells associated with prominent stromal desmoplasia (hematoxylin and eosin stain; magnification, $\mathrm{x} 100)$. Immunohistochemistry revealed positive staining for (C) cytokeratin AE1/AE3 and (D) desmin. Magnification, x400.

This tumor shows a male predilection of $\sim 10: 1$ (8). DSRCT is frequently found in the abdominal cavity, although cases with involvement of the thoracic cavity, paratesticular area, kidney, head and neck region have been reported $(1,8-10)$. The most frequently presenting symptoms of abdominal DSRCT are vague abdominal pain, gradual enlargement of 


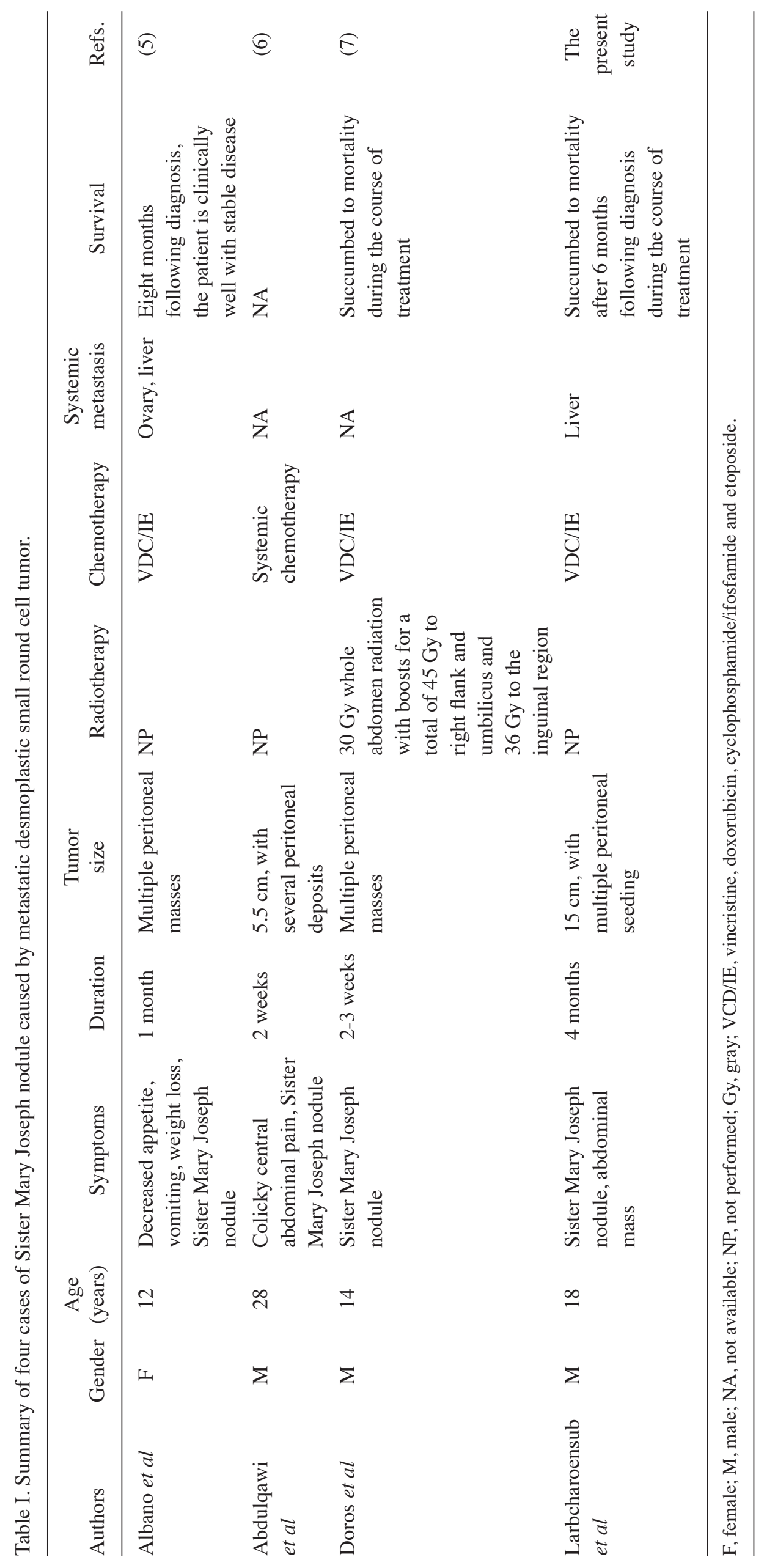


the abdominal mass, abdominal distension, abdominal pain, weight loss and other symptoms associated with obstruction of the intestinal or urinary tract (5-10). The most common site of metastasis is the regional abdominal lymph node. Distant metastases usually involve the lung, liver and bone $(1,8)$. The routine initial laboratory investigations and serum tumor markers are non-contributory. The most useful diagnostic tool is the CT scan, which reveals a characteristic pattern of multiple intra-abdominal masses without any apparent association with the primary organ. FNA of the affected organs may allow early recognition of malignant soft tissue tumors. Core needle or opened biopsy is required for an accurate immunohistopathological diagnosis. Identification and confirmation of the primary tumor is important in order to facilitate treatment.

The macroscopic findings of DSRCT are solid, firm and multilobulated masses, with a gray-white cut surface. The tumor size ranges vary from 1 to $40 \mathrm{~cm}$, with an average size of $10 \mathrm{~cm}(1,8,10)$. The microscopic findings demonstrate solid sheets, large nests, small clumps, or cords of cohesive, small, round, ovoid or spindled cells lying in a hypocellular, desmoplastic, collagenous stroma. The tumor cells are characterized by small, round, oval or elongated hyperchromatic nuclei, clumped chromatin, inconspicuous nucleoli and ill-defined, lightly eosinophilic cytoplasm with an indistinct cytoplasmic border.

The differential diagnoses of primary malignant small round cell tumor of the abdomen include lymphoma, primitive neuroectodermal tumor (PNET)/Ewing sarcoma, small cell carcinoma, rhabdomyosarcoma, neuroblastoma, Wilms' tumor and extrarenal rhabdoid tumor (8-13). Lymphoma histologically reveals atypical lymphocytes infiltrating and replacing normal structure. Negative results of immunohistochemical stains for LCA, CD3 and CD20 may be helpful in excluding lymphoma. The PNET/Ewing sarcoma may be histologically indistinguishable from DSRCT. In the present case study, the immunohistochemical stains were positive for desmin, vimentin, keratin and WT1, which are typically negative for PNET/Ewing sarcoma (10-13). Small cell carcinoma demonstrates many cytological and histological similarities to DSRCT. Clinically, small cell carcinoma is associated with a much older patient population, and usually originates in the lung. On histological examination, a desmoplastic stroma is not identified to be a feature of small cell carcinoma. However, small cell carcinoma demonstrates immunoreactivity with epithelial markers, including cytokeratin and EMA, but is negative for myogenic markers, such as desmin. Rhabdomyosarcoma may reveal small blue cells arranged in nests or sheets. Immunohistochemically, rhabdomyosarcoma is positive for muscle markers, but usually negative for cytokeratin and neural markers, including NSE (6-9). Neuroblastoma and Wilms' tumor also share several histological features with DSRCT, although they occur predominantly in young children and are typically associated with adrenal and renal masses, respectively. Extrarenal rhabdoid tumor is characterized by loss of SMARCB1/INI1, as revealed by immunohistochemistry.

Sister Mary Joseph nodules are rare physical signs that are encountered in $1-3 \%$ of patients with intra-abdominal and/or pelvic malignancy (4). Commonly encountered primary tumors associated with umbilical metastasis include stomach, ovary, endometrium, large intestine and pancreas. The occurrence of a Sister Mary Joseph nodule metastasizing from the DSRCT is very rare. Table I compares the present rare case of DSRCT with three reported cases of Sister Mary Joseph nodule caused by metastatic DSRCT that have been previously described in the literature (5-7). The presence of Sister Mary Joseph nodule often means a poor prognosis, with a median survival time of 6 months for metastatic DSRCT. Metastases to the umbilicus occur predominantly through the lymphatic and venous channels, although contiguous extension from the peritoneal surface and embryonic remnant has been reported (4). In the present case study, the presence of tumor nests on the peritoneal surface and in numerous lymphatic channels indicated that more than one mechanism could have been involved.

Reports of DSRCT have identified reciprocal translocation $(11 ; 22)(\mathrm{p} 13 ; \mathrm{q} 12)$, resulting in a fusion gene between exon 7 of the Ewing sarcoma RNA-binding protein 1 (EWSRl) gene on chromosome 22 and exon 8 of the WT1 suppressor gene on chromosome 11 (1). The resultant chimeric protein is considered to be a transcriptional activator that fails to suppress tumor cell growth. The EWSRI-WT1 chimeric transcript also induces expression of endogenous platelet-derived growth factor-A (PDGFA) (14,15). PDGFA is a potent fibroblastic growth factor that could contribute to one of the most distinctive histological features of DSRCT: The dense fibrous or desmoplastic stroma, consisting of collagen fibers and an important component of elongated mesenchymal cells with features of fibroblasts or myofibroblasts (14). In addition, studies of the EWSR1-WT1 aberrant transcription factor have revealed deregulation of several target genes, including interleukin 2 receptor $\beta(I L-2 R \beta)$, BAI1-associated protein 3 (BAIAP3), myelodysplasia/myeloid leukemia factor 1 (MLF1), T-cell acute lymphoblastic leukemia-associated antigen 1 (TALLA-1) and leucine-rich repeat containing 15 (LRRC15) (15).

The pathogenesis of DSRCT has yet to be fully elucidated. The possibility that DSRCT is of mesothelial origin has been suggested due to the diffuse peritoneal pattern of spread, the presence of epithelial differentiation in the tumor cell, and the fact that fetal mesothelium co-expresses keratin and desmin (16). The small cell mesothelioma also frequently expresses NSE. It has been suggested that DSRCT may be blastomas arising from the lateral mesoderm or intraembryonic coelom (17). Moreover, the serosal lining of body cavities of splanchnic lateral mesoderm, the most common site of DSRCT, has high transient fetal expression of the WT1 gene (15). However, EWSR1-WT1 is typically expressed in tissues derived from the intermediate mesoderm (1). Further molecular study in DSRCT patients is warranted, and has important implications for the study of the pathogenesis of disease.

Radical surgery and adjuvant or neoadjuvant chemotherapy remain the cornerstone of the treatment of DSRCT. However, a complete resection is rarely possible, since DSRCT tends to be large, multifocal and invasive. Several chemotherapy regimens, including alkylating agents and an aggressive chemotherapy regimen followed by myeloablative chemotherapy and autologous stem cell rescue, have been tried, although the result failed to demonstrate a clear benefit for autologous stem cell transplant in improving the clinical outcome (18). Current treatment protocols include multiagent chemotherapy 
and adjuvant surgery and radiotherapy (19). The emphasis is on achieving a complete and durable response. DSRCT has a highly aggressive clinical course, with a high risk of local recurrence and distant metastases. The median survival time is $~ 17-25$ months (18). Improved survival rates are associated with complete resection of the tumor. Poor survival rates, with a median survival time of 6 months, are associated with Sister Mary Joseph nodule caused by metastatic DSRCT (5-7). Until more effective forms of treatment are found, the authors of this case study recommend multimodality treatment, including chemotherapy, surgery and radiotherapy.

In conclusion, DSRCT must be considered in the differential diagnosis of metastatic tumor of the umbilicus forming Sister Mary Joseph nodule. The application of an immunocytohistochemical investigation, in conjunction with the clinical, radiological and histopathological findings, may assist in making the diagnosis, leading to the appropriate treatment.

\section{References}

1. Antonescu CR and Ladanyi M: Desmoplastic small round cell tumour. In: World Health Organization (WHO) Classification of Tumours of Soft tissue and Bone. Pathology and Genetics. Fletcher CDM, Bridge JA, Hogendoorn PCW and Mertens F (eds). Vol 5. 4th edition. IARC Press, Lyon, pp225-pp227, 2013.

2. Gerald WL and Rosai J: Case 2. Desmoplastic small cell tumor with divergent differentiation. Pediatr Pathol 9: 177-183, 1989.

3. Lal DR, Su WT, Wolden SL, Loh KC, Modak S and La Quaglia MP: Results of multimodal treatment for desmoplastic small round cell tumour. J Pediatr Surg 40: 251-255, 2005.

4. Galvan VG: Sister Mary Joseph's nodule. Ann Intern Med 128 410, 1998.

5. Albano EA and Kanter J: Images in clinical medicine. Sister Mary Joseph's nodule. N Engl J Med 352: 1913, 2005.

6. Abdulqawi R, Ahmad S and Ashawesh K: A rare cause of Sister Mary Joseph's nodule. Swiss Med Wkly 137: 559-560, 2007.

7. Doros L, Kaste SC and Rodriguez-Galindo C: Sister Mary Joseph's nodule as presenting sign of a desmoplastic small round cell tumor. Pediatr Blood Cancer 50: 388-390, 2008.
8. Lae ME, Roche PC, Jin L, Lloyd RV and Nascimento AG: Desmoplastic small round cell tumor: A clinicopathologic, immunohistochemical, and molecular study of 32 tumors. Am J Surg Pathol 26: 823-835, 2002

9. Hassan I, Shyyan R, Donohue JH, Edmonson JH, Gunderson LL, Moir CR, Arndt CA, Nascimento AG and Que FG: Intraabdominal desmoplastic small round cell tumors: A diagnostic and therapeutic challenge. Cancer 104: 1264-1270, 2005.

10. Chang F: Desmoplastic small round cell tumors: Cytologic, histologic, and immunohistochemical features. Arch Pathol Lab Med 130: 728-732, 2006

11. Barnoud R, Sabourin JC, Pasquier D, Ranchère D, Bailly C, Terrier-Lacombe MJ and Pasquier B: Immunohistochemical expression of WT1 by desmoplastic small round cell tumor: A comparative study with other small round cell tumors. Am J Surg Pathol 24: 830-836, 2000.

12. Devoe K and Weidner N: Immunohistochemistry of small round-cell tumors. Semin Diagn Pathol 17: 216-224, 2000.

13. Arnold MA, Schoenfield L, Limketkai BN and Arnold CA Diagnostic pitfalls of differentiating desmoplastic small round cell tumor (DSRCT) from Wilms tumor (WT): Overlapping morphologic and immunohistochemical features. Am J Surg Pathol 38: 1220-1226, 2014

14. Lee SB, Kolquist KA, Nichols K, Englert C, Maheswaran S, Ladanyi M, Gerald WL and Haber DA: The EWS-WT1 translocation product induces PDGFA in desmoplastic small round-cell tumour. Nat Genet 17: 309-313, 1997.

15. Goodman KA, Wolden SL, La Quaglia MP and Kushner BH Whole abdominopelvic radiotherapy for desmoplastic small round-cell tumor. Int J Radiat Oncol Biol Phys 54: 170-176, 2002.

16. Hurlimann J: Desmin and neural marker expression in mesothelial cells and mesotheliomas. Hum Pathol 25: 753-757, 1994.

17. Yeoh G, Russell P, Wills EJ and Fleming S: Intra-abdominal desmoplastic small round cell tumor. Pathology 25: 197-202, 1993.

18. Forlenza CJ, Kushner BH, Kernan N, Boulad F, Magnan H, Wexler L, Wolden SL,LaQuaglia MP and Modak S: Myeloablative chemotherapy with autologous stem cell transplant for desmoplastic small round cell tumor. Sarcoma 2015: 269197, 2015.

19. Kallianpur AA, Shukla NK, Deo SV, Yadav P, Mudaly D Yadav R and Palaniappan RM: Updates on the multimodality management of desmoplastic small round cell tumor. J Surg Oncol 105: 617-621, 2012. 